\title{
Analysis of the Relevance of Evaluation Criteria for Multicomponent Image Segmentation
}

\author{
Sié Ouattara ${ }^{1}$, Georges Laussane Loum ${ }^{1}$, Alain Clément ${ }^{2}$, Bertrant Vigouroux ${ }^{2}$ \\ ${ }^{1}$ Laboratoire d'Instrumentation, d'Image et de Spectroscopie (L2IS), Institut National Polytechnique Félix Houphouët-Boigny \\ (INPHB)/DFR-GEE, Yamoussoukro, Côte d'Ivoire; ${ }^{2}$ Laboratoire d'Ingénierie des Systèmes Automatisés (LISA), Institut Univer- \\ sitaire de Technologie, Angers, France. \\ Email: sie_ouat@yahoo.fr
}

Received May $8^{\text {th }}, 2011$; revised June $2^{\text {nd }}, 2011$; accepted June $11^{\text {th }}, 2011$.

\begin{abstract}
Image segmentation is an important stage in many applications such as image, video and computer processing. Generally image interpretation depends on it. The materials and methods used to demonstrate are described. The results are presented and analyzed. Several approaches and algorithms for image segmentation have been developed, but it is difficult to evaluate the efficiency and to make an objective comparison of different segmentation methods. This general problem has been addressed for the evaluation of a segmentation result and the results are available in the literature. In this work, we first presented some criteria of evaluation of segmentation commonly used in image processing with reviews of their models. Then multicomponent synthetic images of known composition are applied to these criteria to explore the operation and evaluate its relevance. The results show that choosing an assessment method depends on the purpose, however the criterion of Zeboudj appears powerful for the evaluation of region segmentations for properly separated classes, on the contrary the criteria of Levine-Nazif and Borsotti are adapted to the methods of classification and permit to build homogeneous regions or classes. The values of the Rosenbeger criterion are generally low and similar, so hard to make a comparison of segmentations with this criterion.
\end{abstract}

Keywords: Quality of Segmentation, Multicomponent Images, Supervised and Unsupervised Evaluation, Synthetic Images, Metric

\section{Introduction}

Segmentation is an essential step in image processing to the extent that it affects the interpretation which will be made of these images in many application areas [1-4]. They are based on different approaches, such as contour, region and texture. Many algorithms have been proposed in recent decades. Given the multitude of proposed methods, the problem of assessing the quality of segmentation becomes paramount.

Originally, the first criteria for evaluating the quality of segmentation were purely subjective: the observer merely to examine different results of segmentation, to decide which the best was according to the objective. It soon becomes necessary to replace this qualitative method by quantitative methods, defining appropriate quality criteria. The first quantitative criteria date from the Nineties (90), but the field remains open, and new criteria appear regularly in literature [5-7].

These criteria are not intended to provide the abso- lute quality of a segmentation: they serve just to compare, using a given criterion, different segmentation algorithms applied to the same image, or to set up an algorithm to adjust its parameters to provide the best result.

It should be noted that classification with a particular criterion, different algorithms of segmentation can be changed if we change the criterion. Similarly, a choice of parameters optimizes a segmentation algorithm, with respect to a given criterion it may be changed if we change the evaluation criterion.

Throughout this paper, the image segment will be denoted $I$. After segmentation, we get an image $I_{s}$, formed with $N_{s}$ regions $S_{i}$, where $i \in\left\{1, \cdots, N_{s}\right\}$, checking the properties:

$$
\left\{\begin{array}{l}
S_{i} \cap S_{j}=\phi \\
\bigcup_{1}^{N_{S}} S_{i}=I
\end{array}\right.
$$


Denoting by $A$ the number of pixels in the image and by $A_{i}$ that of the region $S_{i}$, properties 1 can be written:

$$
A=\operatorname{Card}(I)=\sum_{1}^{N_{s}} S_{i}
$$

One generally agrees to classify the methods of assessing the quality of a segmentation in two categories, corresponding respectively to a supervised evaluation and an unsupervised evaluation $[6,8]$.

\section{Criteria for Evaluating the Region Segmentation}

\subsection{Supervised Evaluation}

The assessment is known as supervised when a reference segmentation $I_{v}$ (also called ground-truth) is defined. This one can be established on a natural image, by one (or more) operator human expert of application domain, using drawing software. A more comfortable case is that of the synthetic images whose ground truth is rigorously accessible.

The evaluation of a segmentation algorithm is then performed by comparing the segmented image $I_{s}$ with the ground-truth $I_{v}$. Denoted by $V_{j}$, where $j \in\left\{1, \cdots, N_{v}\right\}$, the $N_{v}$ regions of the reference image. The question which is asked is that of the measurement of the similarity (or dissimilarity) between the reference segmentation and the algorithmic segmentation.

One possible answer is provided by the method of $\mathrm{Vi}$ net, search recursively the pairs of regions $\left(S_{i}, V_{j}\right)$ with the highest recovery [9]. $T_{i j}$ recovery regions $S_{i}$ and $V_{j}$ is defined by:

$$
T_{i j}=\operatorname{Card}\left(S_{i} \cap V_{j}\right)
$$

It makes it possible to build a table of covering $T\left(I_{s}, I_{v}\right)$, whose elements are the $t_{i j}$. One initially selects in the table the cell corresponding to the maximum $t_{i j}$. The two corresponding regions are then paired. Then one removes in the table the corresponding line and column, and we iterate the operation until all the cells are treated. $K$ couples thus are obtained:

$$
K=\min \left(N_{s}, N_{v}\right)
$$

Each couple presents a covering $t_{k}$, where $k \in\{1, \cdots, K\}$. The measurement of Vinet is then defined by:

$$
\operatorname{VIN}\left(I_{s}, I_{v}\right)=1-\frac{1}{A} \sum_{k=1}^{K} t_{k}
$$

Assuming a perfect recovery, (1) and (5) show that Vinet's criterion takes the value 0 . It tends to 0.5 for minimal recoveries. The method iteratively chosen to match regions is suboptimal; it does not guarantee maximum recovery across all regions.
Other supervised evaluation criteria can be defined [8], taking into account the number and position of the evil segmented pixels, or even their color. They will not be used in this article.

\subsection{Unsupervised Evaluation}

In most cases, we do not have a ground-truth. It is thus necessary to develop calculable evaluation criteria only on the image $I_{s}$, segmented by algorithm. Many criteria, more or less discriminating, have been proposed. They are based on inter-region variability and (or) intra-region uniformity.

Variability or uniformity is measured from the colors of pixels in the image. The color of the pixel $p$ will be denoted $C(p)$, and the average color of a region $R$ of the image will be denoted $\bar{C}(R)$. In the particular case of textured images, texture attributes may also be implemented. They will form the vectors that we denote $G(R)$ when characterized in a region $R$ of the image.

\subsubsection{Intra-Region Uniformity Criterion of Levine and Nazif}

The intra-region uniformity is translated here by the normalized variance of colors inside each region [10]. The colors' average of the region $S_{i}$ is written:

$$
\bar{C}\left(S_{i}\right)=\frac{1}{A_{i}} \sum_{p \in S_{i}} C(p)
$$

The normalized color variance of the component $C_{q}$, on the region $S_{i}$, is expressed:

$$
\sigma_{q}^{2}\left(S_{i}\right)=4 \frac{\frac{1}{A_{i}} \sum_{p \in S_{i}}\left(C_{q}^{2}(p)-\bar{C}_{q}^{2}\left(S_{i}\right)\right)}{\left(\max _{p \in S_{i}}\left[C_{q}(p)\right]-\min _{p \in S_{i}}\left[C_{q}(p)\right]\right)^{2}}
$$

where $\bar{C}_{q}$ denotes the $q$-th component of $\bar{C}$. The total variance of the color on the region $S_{i}$ is written:

$$
\sigma^{2}\left(S_{i}\right)=\sum_{q=1}^{n} \sigma_{q}^{2}\left(S_{i}\right)
$$

Assuming an image with $n$ components.

The criterion of Levine and Nazif is defined by:

$$
\operatorname{LEV}\left(I_{s}\right)=1-\sum_{i=1}^{N_{s}} \sigma^{2}\left(S_{i}\right)
$$

Assuming that all regions are perfectly homogeneous, the test is 1 since the variance of each region (the numerator of the fraction in the (7)) is zero. The criterion decreases in presence of inhomogeneity. An alternative is to weight in the sum on $i$, each region by the number of pixels. 


\subsubsection{Dissimilarity Measure of Liu and Yang}

Let us consider $e_{i}$ the sum of Euclidean distances (dist) between the colors of pixels in the region $S_{i}$ and the average color of $S_{i}$ :

$$
e_{i}=\sum_{p \in S_{i}} \operatorname{dist}\left[C(p), \bar{C}\left(S_{i}\right)\right]
$$

The criterion of Liu and Yang is defined by [11]:

$$
\operatorname{LIU}\left(I_{s}\right)=\frac{1}{1000 \times A} \sqrt{N_{s}} \sum_{i=1}^{N_{s}} \frac{e_{i}^{2}}{\sqrt{A_{i}}}
$$

If all regions are perfectly homogeneous, the criterion is 0 because of $e_{i}$. Contrary to Levine and Nazif's criterion, that of Liu and Yang does not just evaluate intra-region variance: it penalizes the over-segmentation by the presence of many regions in numerator and the area of regions in denominator.

\subsubsection{Criterion of Borsotti}

The criterion of Liu and Yang strives only partially against the over-segmentation. Suppose that it leads to a large number of small regions, all homogeneous: the presence of the factor $e_{i}$ in the (11) gives then $\operatorname{LIU}\left(I_{s}\right)=0$, which is characteristic of a "good" segmentation, this is in contradiction with the decision of over-segmentation as starting hypothesis.

To overcome this drawback, Borsotti proposed a neighboring criterion, penalizing small regions even in the case where they are homogeneous [12]

$$
\operatorname{BOR}\left(I_{s}\right)=\frac{1}{1000 \times A} \sqrt{N_{s}} \sum_{i=1}^{N_{s}}\left[\frac{e_{i}^{2}}{1+\log A_{i}}+\frac{v^{2}\left(A_{i}\right)}{A_{i}^{2}}\right]
$$

where $v\left(A_{i}\right)$ is the number of regions comprising $A_{i}$ pixels. The first term under the summation sign penalizes large inhomogeneous regions, while the second penalizes small regions of the same size, even if they are homogeneous. A "successful" segmentation will be characterized by the criterion of Borsotti close to 0 .

\subsubsection{Inter-Region and Intra-Region Contrast of Zeboudj}

The above criteria are only interested in the intra-region homogeneity. The criterion of Zeboudj takes into account not only the intra-region homogeneity, but also the inter-region contrast, in a neighborhood $W(p)$ of the pixel $p$ [13] .

The contrast $\Delta(p, s)$ between two pixels $p$ and $s$ of an image is proportional to the distance separating the colors of these pixels:

$$
\Delta(p, s)=\frac{\operatorname{dist}[C(p), C(s)]}{d_{\max }}
$$

where $d_{\max }$ is the maximum distance possible in the multicomponent space used.

The contrast $\Delta_{I}\left(S_{i}\right)$ within the region $S_{i}$, and the contrast $\Delta_{E}\left(S_{i}\right)$ between the region $S_{i}$ and the neighboring regions are respectively defined by:

$$
\begin{aligned}
& \Delta_{I}\left(S_{i}\right)=\frac{1}{A_{j}} \sum_{p \in S_{i}} \max \left[\Delta(p, s), s \in W(p) \cap S_{i}\right] \\
& \Delta_{E}\left(S_{i}\right)=\frac{1}{L_{i}} \sum_{p \in F_{i}} \max \left[\Delta(p, s), s \in W(p) \cap S_{i}, s \notin S_{i}\right]
\end{aligned}
$$

where $L_{i}$ is the length of the boundary $F_{i}$ delimiting the region $S_{i}$. The global contrast $\Delta\left(S_{i}\right)$ of the region $S_{i}$ is defined by:

$$
\Delta\left(S_{i}\right)=\left\{\begin{array}{l}
1-\frac{\Delta_{I}\left(S_{i}\right)}{\Delta_{E}\left(S_{i}\right)} \quad \text { if } 0<\Delta_{I}\left(S_{i}\right)<\Delta_{E}\left(S_{i}\right) \\
\Delta_{E}\left(S_{i}\right) \text { if } \Delta_{I}\left(S_{i}\right)=0 \\
0 \quad \text { else if }
\end{array}\right.
$$

Zeboudj's criterion is deduced by:

$$
\operatorname{ZEB}\left(I_{s}\right)=\frac{1}{A} \sum_{i=1}^{N_{s}} A_{i} \times \Delta\left(S_{i}\right)
$$

This criterion increases with the quality of segmentation. It is not suitable for images too noisy or textured.

\subsubsection{Criterion of Rosenberger}

To take into account the possible presence of textured regions in the segmented image, while addressing the inter-region disparity, Rosenberger offers a different treatment of textured regions and non-textured regions in the defining of the evaluation criterion of the segmentation [14]. The textured character or not of a region is established using the co-occurrence matrices (thus performing the pre-processing of multicomponent images into scalar images, which is simplified by a process of multiple thresholding in order to reduce the size of co-occurrence matrices). The non-textured regions are characterized by their colors $C\left(p \in S_{i}\right)$, and textured regions by a vector of 29 texture attributes, noted $G$, chosen, because of their discriminating power among the classic attributes of co-occurrence, lengths of intervals, and local histograms of local extremas.

\section{- Intra-region disparity}

The disparity of non-textured regions is characterized by the intra-region variance of pixel colors in a manner analogous to that used by Levine and Nazif. In the region $S_{i}$ attributed the disparity coefficient: 


$$
D\left(S_{i}\right)=\sigma\left(S_{i}\right)
$$

calculated using Equations (6) to (8).

For the textured regions, the attributes used are not any more the colors, but the vectors of texture $G\left(S_{i}\right)$. Their calculation of the coefficient of disparity $D_{I}\left(S_{i}\right)$ associated with textured regions is more complex, and will not be detailed here, because all the images used in the continuation of this work will be considered non-textured. The interested reader may however see reference [14].

The global intra-region disparity is given by:

$$
D_{I}\left(I_{s}\right)=\frac{1}{N_{s}} \sum_{i=1}^{N_{s}} \frac{A_{i}}{A} D_{I}\left(S_{i}\right)
$$

\section{- Inter-region disparity}

Between two non-textured regions, the disparity is proportional to the distance separating the average colors of the two regions:

$$
D\left(S_{i}, S_{j}\right)=\frac{\operatorname{dist}\left[\bar{C}\left(S_{i}\right), \bar{C}\left(S_{j}\right)\right]}{d_{\max }}
$$

where $d_{\max }$ is the maximum distance possible in the multicomponent space used.

Between two textured regions, disparity takes into account the textural distance vectors, and their norm.

$$
D\left(S_{i}, S_{j}\right)=\frac{d\left[G\left(S_{i}\right), G\left(S_{j}\right)\right]}{\left\|G\left(S_{i}\right)\right\|+\left\|G\left(S_{j}\right)\right\|}
$$

Between two regions one of which is textured and the other not, the disparity is set to:

$$
D\left(S_{i}, S_{j}\right)=1
$$

If we denote $\left\{S_{i 1}, \cdots, S_{i q_{i}}\right\}$ the set of $q_{i}$ neighboring regions of $S_{i}$. The inter-region disparity of the region $S_{i}$ is written:

$$
D_{E}\left(S_{i}\right)=\frac{1}{q_{i}} \sum_{j=i 1}^{i_{i}} D\left(S_{i}, S_{j}\right)
$$

and the global inter-region disparity of the image $I_{s}$ is given by:

$$
D_{E}\left(I_{s}\right)=\frac{1}{N_{s}} \sum_{i=1}^{N_{s}} \frac{A_{i}}{A} D_{E}\left(S_{i}\right)
$$

\section{- Criterion of Rosenberger}

Finally, the criterion of Rosenberger is defined by:

$$
\operatorname{ROS}\left(I_{s}\right)=\frac{D_{I}\left(I_{s}\right)+1-D_{E}\left(I_{s}\right)}{2}
$$

This criterion decreases when the segmentation quality increases. A sub-segmentation is penalized through an intra-region disparity $D_{I}$ strong, and over-segmentation will be through an inter-region disparity $D_{E}$ low.

\section{Analysis of Unsupervised Evaluation Criteria, Results and Discussion}

The behavior of the above criteria will be studied using synthetic images constituted of regions of uniform color, perfectly controlled: their segmentation in region is thus known. The segmented image and the ground-truth being rigorously similar, it is unnecessary to examine the behavior of Vinet's criterion, which will systematically give a result equal to 0 (perfect overlap). Only then we will study the unsupervised evaluation criteria (except that of Liu and Yang: we have preferred to him that of Borsotti, which is an improvement). The window $W$ used to calculate Zeboudj's criterion here is a window of size $3 \times 3$ (neighboring order 8 ). To calculate Rosenberger's criterion, all regions will be considered non-textured.

\subsection{Characteristics of Synthetic Images Used}

The test images are 4 synthetic images shown in Figure 1 and whose detailed composition is described in Table 1. Their segmentation (shown in false color to better highlight the regions) is shown in Figure 2.

The first two images (Syntla_Lisa and Synt1b_Lisa) present two uniform regions, of different sizes. The colors of these regions are clearly separated for the image Synt1a_Lisa, and otherwise very similar (and indistinguishable to the eye) for the image Synt1b_Lisa. The last two images (Synt2a_Lisa and Synt2b_Lisa) present three

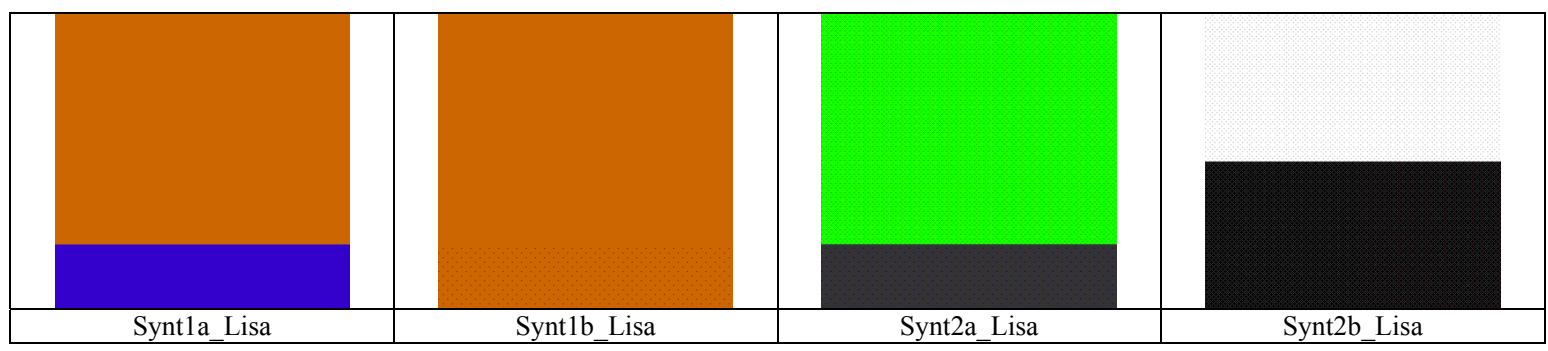

Figure 1. Synthetic images. 


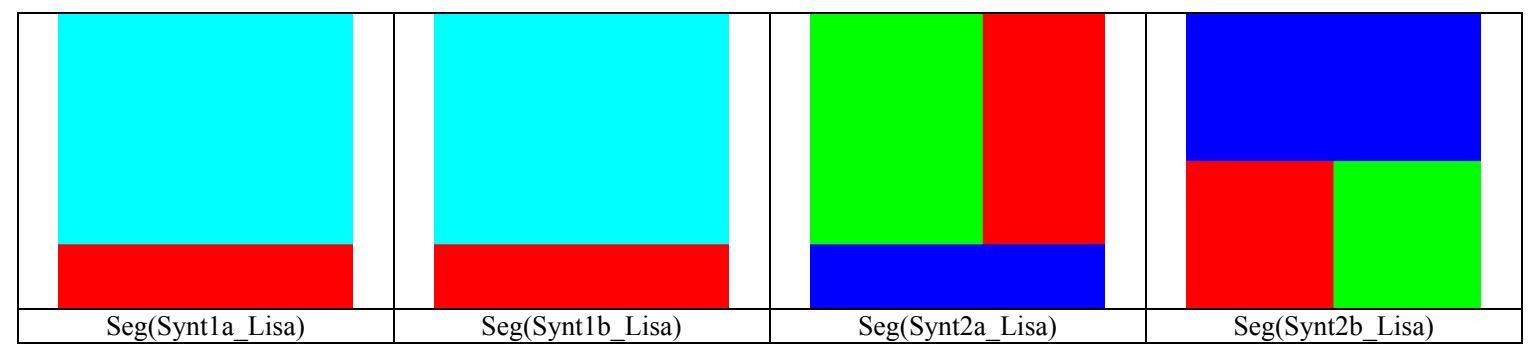

Figure 2. Result (in arbitrary colors) of the segmentation.

Table 1. Building of synthetic images of Figure 1.

(a)

\begin{tabular}{cccccccc}
\hline \multicolumn{1}{c}{ Building of synthetic images } \\
\hline \multicolumn{4}{c}{ Synt1a_Lisa } & \multicolumn{4}{c}{ Synt1b_Lisa } \\
$R$ & $G$ & $B$ & Population & $R$ & $V$ & $B$ & Population \\
51 & 0 & 204 & 51200 & 203 & 101 & 0 & 14336 \\
204 & 102 & 0 & 14336 & 204 & 102 & 0 & 51200 \\
\hline
\end{tabular}

(b)

\begin{tabular}{cccccccc}
\hline \multicolumn{1}{c}{ Building of synthetic images } \\
\hline \multicolumn{1}{c}{ Synt2a_Lisa } & \multicolumn{4}{c}{ Synt2b_Lisa } \\
$R$ & $G$ & $B$ & Population & $R$ & $V$ & $B$ & Population \\
21 & 250 & 0 & 21200 & 20 & 20 & 20 & 16384 \\
23 & 250 & 0 & 30000 & 22 & 20 & 20 & 16384 \\
51 & 50 & 54 & 14336 & 250 & 250 & 250 & 32768 \\
\hline
\end{tabular}

uniform regions.

On the Synt2a Lisa image, two regions have neighboring colors (and indistinguishable with the eye) and Different sizes, higher than that of the third region, from which the color is distant. On the Synt2b_Lisa image, two regions are identical in size, equal to half of that of the third region: the colors of the two regions of identical size are very close one to the other (indiscernible to the eye), and far away from the color of the third.

\subsection{Calculation and Analysis of Criteria Evaluation for Segmentation}

The evaluation criteria for unsupervised segmentation, computed for these images, take the values reported in Table 2.

Each region is of uniform color, Levine's criterion returns the possible maximum value 1, as expected.

For the same reason, Borsotti's test is sensitive only to the size of regions and the number of regions with the same size (second term under the summation sign in (12)). Segmentation provides a partition exactly the same for the first two images, and consequently, the criteria of Borsotti are identical in both cases. Compared to the first two images, the latter two are penalized by the criterion of Borsotti (which increases), because the number of regions increases from 2 to 3 . The fourth image is most penalized than the third because it has two regions of identical size (parameter $v$ of Borsotti's criterion).
Table 2. Value of evaluation criteria for unsupervised segmentation for synthetic images of Figure 1.

\begin{tabular}{ccccc}
\hline & \multicolumn{4}{c}{ Criterion } \\
\cline { 2 - 5 } Image & $\begin{array}{c}\text { Levine } \\
\text { and } \\
\text { Nazif }\end{array}$ & Borsotti & Zeboudj & Rosenberger \\
\hline Synt1a_Lisa & 1 & $1,13 \cdot 10^{-16}$ & 0,6000 & 0,3500 \\
Synt1b_Lisa & 1 & $1,13 \cdot 10^{-16}$ & 0,0026 & 0,4993 \\
Synt2a_Lisa & 1 & $2,17 \cdot 10^{-16}$ & 0,1957 & 0,4584 \\
Synt2b_Lisa & 1 & $8,12.10^{-16}$ & 0,6753 & 0,3873 \\
\hline
\end{tabular}

As each region is uniform in color, the internal contrast of regions $\Delta_{I}$ is zero, and Zeboudj criterion no longer considers the contrast between a region and its neighbors. It penalizes the image Synt1b_Lisa $(Z E B=$ 0.0026) compared to the image Syntla_Lisa $(Z E B=$ 0.6000). The same reasoning, with the same conclusions can be made with the criterion of Rosenberger.

\subsection{Influence of Inter-Class Colorimetric Distance and the Population of Regions}

Consider a square image of $a$ hand consisting of two homogeneous regions of color respective $C_{1}$ and $C_{2}$ which $n$ components are coded from levels 0 to $2^{q}-1$. The position of the boundary between the two regions is marked by a variable $x$ (Figure 3 ) proportional to the population of both regions.

We find generalize the model of images Synt1a_Lisa and Synt1b_Lisa of Figure 1. The calculation of the evaluation criteria for segmentation provides here the following results:

$$
L E V=1
$$

$$
\begin{gathered}
B O R=\left\{\begin{array}{lr}
\frac{\sqrt{2}}{1000 a^{6}}\left[\frac{1}{x^{2}}+\frac{1}{(1-x)^{2}}\right] & \text { if } \quad x \neq \frac{1}{2} \\
\frac{32 \sqrt{2}}{1000 a^{6}} & \text { if } \quad x=\frac{1}{2}
\end{array}\right. \\
Z E B=\frac{1}{a} \frac{\operatorname{dist}\left(C_{1}, C_{2}\right)}{d_{\max }}
\end{gathered}
$$




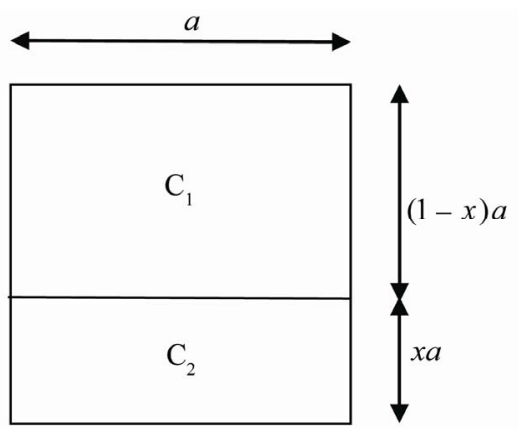

Figure 3. Square image consisting of two regions of color $\mathrm{C}_{1}$ and $\mathrm{C}_{2}$.

$$
R O S=\frac{2-\frac{\operatorname{dist}\left(C_{1}, C_{2}\right)}{d_{\max }}}{8}
$$

The Equation (26) shows that Levine's criterion values the segmentation into homogeneous regions, regardless of their sizes and colors. Segmented into two neighboring regions of nearly identical color will be as good as if the colors were distant, which may be irrelevant for the intended application.

The function between brackets in (27) is decreasing in the interval $[0,0.5]$ and increasing in the interval $[0.5,1]$. This shows that Borsotti's criterion values the segmentation in homogeneous regions of similar size, regardless of their spacing colors. A segmentation into two neighboring regions of very different sizes will be considered bad, even if the application is to detect a small homogeneous region within a greater one.

Equations (28) and (29) show that Zeboudj's and Rosenberger's criteria value the segmentations into two neighboring homogeneous regions in proportion to their color difference, and this result holds true regardless of the sizes of the two regions.

\subsection{Influence of the Metric on Zeboudj's and Rosenberger's Criteria}

Denote $Z E B_{\min }$ and $Z E B_{\max }$ (respectively $R O S_{\min i}$ and $R O S_{\max }$ ) limits of variation of Zeboudj parameter (respectively that of Rosenberger) for an image which model is that of Figure 3. The minimum distance between two colors is equal to 1, whether we use the Euclidean metric, that of Manhattan, or that of Chebychev. Since the $n$ components of the image are each coded on $q$ bits, the maximum distance between two colors is written:

$$
d_{\max }= \begin{cases}\sqrt{n \times 2^{2 q}} & \text { in Euclidian metric } \\ n \times 2^{q} & \text { in Manhattan metric } \\ 2^{q} & \text { in Chebychev metric }\end{cases}
$$

The range of variation of Zeboudj and Rosenberger criteria that results is summarized in Table 3 , for grayscale images $(n=1)$, color images $(n=3)$ and multicomponent images $(n=10)$, the tonal resolution $q$ can vary from 1 to 8 bits per component. $Z E B_{\max }$ and $R O S_{\text {mini }}$, who write the best segmentations, are not listed in the table because they are respectively 1 and 0.125 for all configurations.

For scalar images $(n=1)$, the values of $Z E B_{\min }$ and $R O S_{\text {max }}$ repeated identically whatever the type of metric, which is not surprising since the distances are $d_{\max }$ identical.

For all metrics, the value of $2 \times Z E B_{\min }$ characteristic of bad segmentation is less than 0.1 regardless of the number of components, provided that the tonal resolution is greater than or equal to 4 bits. This provides ranges of variation in a width at least equal to 0.9 (for 4 bits per pixel), which approaches the limit 1 when the tonal resolution increases. It is therefore possible in this case to use the metric of choice, without significant impact on the available scale of classification of segmentation algorithms.

We will have an incentive to choose Chebychev's metric, which induces less computation. At lower tonal resolutions, the range of possible values for $2 \times Z E B$ shrinks, until a width equal to 0.5 for binary images. But again this difference remains the same whatever the metric used.

For Chebychev's metric, the maximum distance between two colors do not depend on the number of components (see (30)). This explains the identical repetition of values of $Z E B_{\min }$ and $R O S_{\max }$, whatever the number of components (two last columns of Table 3).

For all metrics, the value of $R O S_{\max }$ characteristic of poor segmentation is between 0.240 and 0.250 regardless of the number of components, as long as the tonal resolution is greater than 4 bits. This provides ranges of variation in a width at least equal to 0.115 (for 4 bits per pixel), which tends to the limit 0.125 when increases tonal resolution. Again it will be possible to use the metric of choice. At lower tonal resolutions, the range of possible values for ROS narrows to a width equal to 0.0625 for binary images. But again this difference remains the same whatever the metric used.

\section{Conclusions}

This work has enabled us to study the unsupervised evaluation methods of segmentation to understand their relevance. We showed that their relevance is related to the metric used, the distance between color regions or classes, classes or regions size, the tonal resolution of images and the number of components $n$ of the images without forgetting the homogeneity of the regions. We 
Table 3. Value of evaluation Variation range of Zeboudj and Rosenberger criteria, for images consistent with the model in Figure 3 ( $a / 2$ was assumed equal to 1 for the calculation of the Zeboudj criterion).

\begin{tabular}{|c|c|c|c|c|c|c|c|}
\hline \multirow[b]{2}{*}{$n$} & \multirow[b]{2}{*}{$q$} & \multicolumn{2}{|c|}{ Euclidean distance } & \multicolumn{2}{|c|}{ Manhattan distance } & \multicolumn{2}{|c|}{ Chebychev distance } \\
\hline & & $2 * Z E B_{\min }$ & $R O S_{\max }$ & $2 * Z E B_{\min }$ & $R O S_{\max }$ & $2 * Z E B_{\min }$ & $R O S_{\max }$ \\
\hline \multirow{6}{*}{1} & 1 & 0.500 & 0.188 & 0.500 & 0.188 & 0.500 & 0.188 \\
\hline & 3 & 0.125 & 0.234 & 0.125 & 0.234 & 0.125 & 0.23 \\
\hline & 4 & 0.063 & 0.242 & 0.063 & 0.242 & 0.063 & 0.242 \\
\hline & 5 & 0.031 & 0.246 & 0.031 & 0.246 & 0.031 & 0.246 \\
\hline & 7 & 0.008 & 0.249 & 0.008 & 0.249 & 0.008 & 0.249 \\
\hline & 8 & 0.004 & 0.250 & 0.004 & 0.250 & 0.004 & 0.250 \\
\hline \multirow{7}{*}{3} & 1 & 0.289 & 0.214 & 0.167 & 0.229 & 0.500 & 0.188 \\
\hline & 2 & 0.144 & 0.232 & 0.083 & 0.240 & 0.250 & 0.219 \\
\hline & 3 & 0.072 & 0.241 & 0.042 & 0.245 & 0.125 & 0.234 \\
\hline & 4 & 0.036 & 0.245 & 0.021 & 0.247 & 0.063 & 0.242 \\
\hline & 5 & 0.018 & 0.248 & 0.010 & 0.249 & 0.031 & 0.246 \\
\hline & 7 & 0.005 & 0.249 & 0.003 & 0.250 & 0.008 & 0.249 \\
\hline & 8 & 0.002 & 0.250 & 0.001 & 0.250 & 0.004 & 0.250 \\
\hline \multirow{8}{*}{10} & 1 & 0.158 & 0.230 & 0.050 & 0.244 & 0.500 & 0.188 \\
\hline & 2 & 0.079 & 0.240 & 0.025 & 0.247 & 0.250 & 0.219 \\
\hline & 3 & 0.040 & 0.245 & 0.013 & 0.248 & 0.125 & 0.234 \\
\hline & 4 & 0.020 & 0.248 & 0.006 & 0.249 & 0.063 & 0.242 \\
\hline & 5 & 0.010 & 0.249 & 0.003 & 0.250 & 0.031 & 0.246 \\
\hline & 6 & 0.005 & 0.249 & 0.002 & 0.250 & 0.016 & 0.248 \\
\hline & 7 & 0.002 & 0.250 & 0.001 & 0.250 & 0.008 & 0.249 \\
\hline & 8 & 0.001 & 0.250 & 0.000 & 0.250 & 0.004 & 0.250 \\
\hline
\end{tabular}

also showed what ranges of evaluation criteria were good or bad.

Knowing that the study of the relevance of a segmentation algorithm requires choosing an evaluation criterion of segmentation, therefore to realize the goal of the segmentation in homogeneous regions and well separated, we recommend an appropriate combination of Zeboudj's and Borsotti's criteria.

In Perspectives, an experiment with models of synthetic multi-region images will achieve a comprehensive study of the relevance of unsupervised evaluation methods of segmentation.

\section{REFERENCES}

[1] W. Wei-Yi, L. Zhan-Ming, Z. Gui-Cang and Z. GuoQuan, "Novel Color Microscopic Image Segmentation with Simultaneous Uneven Illumination Estimation Based on PCA," Information Technology Journal, Vol. 9, No. 8, 2010, pp. 1682-1685.doi: 10.3923/itj.2010.1682.1685

[2] A. Mohammadzadeh, M. J. Valadan Zoej and A. Tavakoli, "Automatic Main Road Extraction from High Resolution Satellite Imageries by Means of Self-Learning Fuzzy-ga Algorithm," Journal of Applied Sciences, Vol. 8, No. 19, 2008, pp. 3431-3438. doi: 10.3923/jas.2008.3431.3438

[3] J. Freixenet, X. Munoz, D. Raba, J. Marti and X. Cufi, "Yet Another Survey on image Segmentation: Region and Boundary Information Integration," Lecture Notes in
Computer Science, Vol. 2352, 2002, pp. 21-25.

[4] R. M. Haralick and L. G. Shapiro, "Survey: Image Segmentation Techniques," Computer Vision, Graphics and Image Processing, Vol. 29, No. 1, 1985, pp. 100-132. doi: $10.1016 / \mathrm{s} 0734-189 \mathrm{x}(85) 90153-7$

[5] H. Zhang, J. E. Fritts and S. A. Goldman, "Image Segmentation Evaluation: A Survey of Unsupervised Methods," Computer Vision and Image Understanding, Vol. 110, No. 2, 2008, pp. 260-280. doi: 10.1016/j.cviu.2007.08.003

[6] S. Chabrier, B. Emile, C. Rosenberger and H. Laurent, "Unsupervised Performance Evaluation of Image Segmentation, Special Issue on Performance Evaluation in Image Processing," EURASIP Journal on Applied Signal Processing, Vol. 2006, 2006, pp. 1-12. doi: 10.1155/ASP/2006/96306

[7] J-P. Coquerez and S. Philipp, "Image Analysis: Filtering and Segmentation," Masson Edition, Paris, 1995.

[8] S. Philipp-Foliguet and L. Guigues, "Evaluation of Segmentation: State of the Art, New Indices and Comparison," Signal Processing, Vol. 23, No. 2, 2006, pp. 109124. doi: $10.4267 / 2042 / 5824$

[9] L. Vinet, "Segmentation and Mapping of Areas of Stereoscopic Pairs of Images," Ph.D. Thesis, University of Paris IX Dauphine, Paris, 1991.

[10] M. D. Levine and A. M. Nazif, "Dynamic Measurement of Computer Generated Image Segmentations," IEEE Transactions on Pattern Analysis and Machine Intelli- 
gence, Vol. 7, No. 2, 1985, pp. 155-164. doi: 10.1109/TPAMI.1985.4767640

[11] J. Liu and Y.-H. Yang, "Multiresolution Color Image Segmentation," IEEE Transactions on PAMI, Vol. 16, No. 7, 1994, pp. 689-700. doi: 10.1109/34.297949

[12] M. Borsotti, P. Campadelli and R. Schettini, "Quantitative Evaluation of Color Image Segmentation Results," Pattern Recognition Letters, Vol. 19, No. 8, 1998, pp. 741-747. doi: 10.1016/S0167-8655(98)00052-X
[13] R. Zeboudj, "Filtering, Automatic Thresholding, Contrast and Contours: The Pre-Treatment with the Image Analysis," Ph.D. Thesis, University of Saint Etienne, Saint Etienne, 1988.

[14] C. Rosenberger, "Adaptative Evaluation of Image Segmentation Results," 18th International Conference on Pattern Recognition, Vol. 2, 2006, pp. 399-402.

doi: 10.1109/ICPR.2006.214 